\title{
ELMs and the H-Mode Pedestal in NSTX
}

R. Maingia, S.A. Sabbagh, C.E. Busa, E.D. Fredrickson, J.E. Menard, D. Stutman, K. Tritz, M.G. Bell, R.E. Bell, J.A. Boedo, D.A. Gates, D.W. Johnson, R. Kaita, S.M. Kaye, H.W. Kugel, B.P. LeBlanc, D. Mueller, R. Raman, A.L. Roquemore, V.A. Soukhanovskii, T. Stevenson

This article was submitted to the $16^{\text {th }}$ International Conference on Plasma Surface Interactions, Portland, Maine, May 24-28, 2004

\section{May 2004}




\section{DISCLAIMER}

This document was prepared as an account of work sponsored by an agency of the United States Government. Neither the United States Government nor the University of California nor any of their employees, makes any warranty, express or implied, or assumes any legal liability or responsibility for the accuracy, completeness, or usefulness of any information, apparatus, product, or process disclosed, or represents that its use would not infringe privately owned rights. Reference herein to any specific commercial product, process, or service by trade name, trademark, manufacturer, or otherwise, does not necessarily constitute or imply its endorsement, recommendation, or favoring by the United States Government or the University of California. The views and opinions of authors expressed herein do not necessarily state or reflect those of the United States Government or the University of California, and shall not be used for advertising or product endorsement purposes.

This is a preprint of a paper intended for publication in a journal or proceedings. Since changes may be made before publication, this preprint is made available with the understanding that it will not be cited or reproduced without the permission of the author.

This report has been reproduced directly from the best available copy.

Available to DOE and DOE contractors from the

Office of Scientific and Technical Information

P.O. Box 62, Oak Ridge, TN 37831

Prices available from (423) 576-8401

http: / / apollo.osti.gov/bridge/

Available to the public from the National Technical Information Service

U.S. Department of Commerce 5285 Port Royal Rd., Springfield, VA 22161 http:/ / www.ntis.gov/

OR

Lawrence Livermore National Laboratory Technical Information Department's Digital Library http:/ / www.llnl.gov/tid/Library.html 


\section{ELMs and the H-mode Pedestal in NSTX}

R. Maingi ${ }^{a}$, S.A. Sabbagh ${ }^{\text {b }}$, C.E. Bush ${ }^{\mathrm{a}}$, E.D. Fredrickson ${ }^{\mathrm{c}}$, J.E. Menard ${ }^{\mathrm{c}}$, D. Stutman ${ }^{\mathrm{d}}$, K. Tritz ${ }^{\mathrm{d}}$, M.G. Bell' , R.E. Bell ${ }^{c}$, J.A. Boedo ${ }^{\mathrm{e}}$, D.A. Gates ${ }^{\mathrm{c}}$, D.W. Johnson ${ }^{\mathrm{c}}$, R. Kaita ${ }^{\mathrm{c}}$, S.M. Kaye ${ }^{\mathrm{c}}$, H.W.

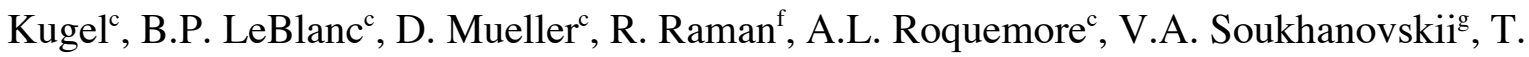
Stevenson $^{\mathrm{c}}$

${ }^{a}$ Oak Ridge National Laboratory, Oak Ridge TN, 37831 USA

${ }^{b}$ Columbia University, New York, NY, USA

${ }^{c}$ Princeton Plasma Physics Laboratory, PO Box 451, Princeton, NJ, 08543 USA

dJohns Hopkins University, Baltimore, MD, USA

${ }^{e}$ University of California San Diego, San Diego CA USA

${ }^{f}$ University of Washington, Seattle WA, USA

${ }^{g}$ Lawrence Livermore National Lab, Livermore CA USA

\section{$\underline{\text { Abstract }}$}

We report on the behavior of ELMs in NBI-heated H-mode plasmas in NSTX. It is observed that the size of Type I ELMs, characterized by the change in plasma energy, decreases with increasing density, as observed at conventional aspect ratio. It is also observed that the Type I ELM size decreases as the plasma equilibrium is shifted from a symmetric double-null toward a lower single-null configuration. Type III ELMs have also been observed in NSTX, as well as a high-performance regime with small ELMs which we designate Type V. These Type V ELMs are consistent with high bootstrap current operation and density approaching Greenwald scaling. The Type V ELMs are characterized by an intermittent $n=1$ MHD mode rotating counter to the plasma current. Without active pumping, the density rises continuously through the Type V phase. However, efficient in-vessel pumping should allow density control, based on particle containment time estimates.

JNM keywords: Plasma-materials interaction, Plasma Properties

PSI16 keywords: ELM, Edge pedestal, Fueling, NSTX, Particle control

PACs: $52.55 \mathrm{Fa}$

* corresponding author address: Princeton Plasma Physics Lab, Receiving 3, Route 1 North, Princeton NJ 08543

* corresponding author email: rmaingi@pppl.gov

* presenting author: R. Maingi, email: rmaingi@pppl.gov 


\section{Introduction}

Edge localized modes (ELMs) have been observed in tokamaks since the discovery of the Hmode. ELMs prevent build-up of fuel and impurity particles by periodic ejection of the edge plasma into the scrape-off layer and divertor plasma, where they may be exhausted. This periodic ejection results in transiently high power and particle loads to the plasma facing components (PFC). Nonetheless, the baseline operating scenario for the International Thermonuclear Experimental Reactor (ITER) relies on H-Mode confinement and profiles ${ }^{1}$, partly because the best plasma performance is often realized in the so-called Type I ELM regime. The Type I ELM typically results in larger heat pulses than other ELM types ${ }^{2}$. Such transient PFC loading is tolerable in present day machines, but extrapolations show that severe PFC damage may occur in larger, higher power density machines (such as ITER) when the ELM power loading exceeds material limits. Thus the scaling of power loss to PFCs during Type I ELMs, as well as the search for smaller/no ELM regimes with good performance, has received significant attention in fusion research. A wide variety of ELMs has been observed in the National Spherical Torus Experiment (NSTX), including Type I, Type III, and a new small ELM regime which we label as 'Type V'. In the remainder of this section and the next, the ELM observations and the results of an experiment to study Type I ELM scaling will be described. Type V ELM characteristics will be described in section III.

The NSTX is a medium-sized, low-aspect ratio spherical tokamak ${ }^{3}$ with the following parameters: major radius $\mathrm{R}=0.85 \mathrm{~m}$, minor radius $\mathrm{a}=0.67 \mathrm{~m}, \mathrm{R} / \mathrm{a} \geq 1.26$, toroidal field $\mathrm{B}_{\mathrm{t}} \square 0.6 \mathrm{~T}$, with up to $7 \mathrm{MW}$ of neutral beam injection $(\mathrm{NBI})$ power $\left(\mathrm{P}_{\mathrm{NBI}}\right)$ and $6 \mathrm{MW}$ of radio-frequency heating. Fig. 1 shows a survey of ELM types in NSTX, along with the corresponding impact on the plasma stored energy. Panel (a) shows Type I ELMs, which reduce the plasma stored energy 
by up to $10 \%$. Panel (b) shows Type III ELMs, which decrease the stored energy by 1-3\%. The Type III ELMs are identified by their inverse heating power/frequency relationship, their occurrence close to the L-H power threshold, and the presence of an electromagnetic pre-cursor oscillation. Panel (c) shows a regime characterized by tiny, rapid ELMs which we designate as Type $\mathrm{V}^{4}$; each ELM has an indiscernible impact on stored energy, although the particle and energy confinement in this Type V ELM regime are reduced compared with ELM-free discharges. Panel (d) shows a mixed ELM regime with Type V ELMs and a giant event, which can reduce the stored energy by $30 \%$. The giant events are sometimes seen under low recycling conditions (e.g. see the next section) and are independent from the Type V ELMs.

\section{ELM size and frequency scaling experiments}

The ELM size and frequency scaling experiments were conducted in diverted discharges with $\mathrm{I}_{\mathrm{p}}=0.9 \mathrm{MA}, \mathrm{B}_{\mathrm{t}}=0.45 \mathrm{~T}$, and elongation $\square \sim 1.9$. At the time of these experiments, high triangularity $(\square \sim 0.8)$ double-null discharges were studied because Type I ELMs were readily obtained $^{5}$. The size of the ELM was computed from fast EFIT reconstructions ${ }^{6,7}$ with 0.25 ms resolution. Here ELM size is measured by the change in stored energy ( $\square \mathrm{W})$ due to the ELM, relative to both the initial stored energy $\mathrm{W}_{0}$ and the pedestal stored energy $\mathrm{W}_{\text {ped. }}$. The pedestal pressure was obtained by fitting the electron pressure profile with a ('standard') modified hyperbolic tangent. The pedestal stored energy was obtained by $\mathrm{W}_{\mathrm{ped}}=0.92 * 3 * \mathrm{P}_{\mathrm{e}, \mathrm{ped}} * \mathrm{~V}_{\mathrm{p}}$, as used for multi-machine scalings ${ }^{8}$. Here $\mathrm{P}_{\mathrm{e} \text {,ped }}$ is the fitted electron pedestal pressure and $\mathrm{V}_{\mathrm{pl}}$ is the plasma volume. Typically the pedestal stored energy fraction $\mathrm{W}_{\mathrm{ped}} / \mathrm{W}_{0}$ lies between $25 \%$ and $33 \%$. We also note that the pedestal stored energy computed with the above equation for NSTX usually lies within $15 \%$ of the value predicted by the recent multi-machine scaling ${ }^{8}$. 
In principle, this technique allows resolution of ELMs up to $\sim 1 \mathrm{kHz}$ frequency, but practically the size becomes limited both by statistics (+/-1-1.5\%) and by the eddy current model used in the present reconstructions, the latter becoming more unreliable as the speed of the transients increases. Thus the ELM size scaling for frequencies above $300 \mathrm{~Hz}$ should be viewed only as an indication of the trend.

\section{II.1. Double-null divertor configurations}

One feature prevalent in most NSTX H-mode discharges with NBI heating is a secular density ramp, due partly to good particle confinement time and partly to continuous fueling by both the neutral beams themselves and by gas injected from the center stack midplane. This center-stack fueling was found to facilitate reproducible H-mode access ${ }^{9,10}$, but the limited space there prevents installation of a fast time response control valve. Hence the center stack gas injector has a $0.55 \mathrm{sec}$ e-folding decay time for the flow rate. Fig. 2 shows the discharge characteristics for a fueling rate scan obtained by using different fill pressures on the center stack midplane injector. It is observed that the density rate of rise increased modestly with the flow rate. At the lowest flow rate, a clear H-mode transition could not be obtained, although there were sometimes dithering transitions in and out of $\mathrm{H}$-mode. It can also be seen that the $\mathrm{D}_{\square}$ modulations due to the ELMs decreased with increasing density. Fig. 3(a-e) shows the results of the ELM size analysis for all ELMs in the fueling rate scan. There is a large variation in the ELM size, but the envelope shrinks with increasing density. In other words, the maximum observed size drops with density as observed at conventional aspect ratio ${ }^{11}$. The maximum stored energy loss fraction $\square \mathrm{W} / \mathrm{W}_{0}$ in this scan approached $7 \%$, and the pedestal loss fraction $\square \mathrm{W} / \mathrm{W}_{\text {ped }}$ reached just above $25 \%$. The 
frequency of the ELMs generally increased with density. Fig. 3e shows that the ELM size generally decreased as the frequency increased. We note that the average ELM size at each fueling rate did not scale with the fueling rate itself, i.e. the density dependence was the primary factor affecting ELM size and frequency.

Fig. 3f shows the average ELM size observed during a divertor configuration scan. Here the change in configuration was characterized by the parameter drsep, defined as the distance at the outer midplane between the the separatrix flux surfaces passing through the upper and lower Xpoints, with the convention that a positive drsep favors the lower divertor. Although the scan is sparse, it does appear that the average ELM size is reduced somewhere between a balanced double-null (drsep=0) and a 'true' lower single-null, e.g. with $d r s e p>$ the ion poloidal gyroradius ( $\sim 1 \mathrm{~cm}$ for these conditions). While these preliminary results require confirmation with a finer $d r s e p$ scan, it is notable that similar results were reported on ASDEX-Upgrade, along with the assertion $^{12}$ that the ELM type became grassy or Type II. We also note that drsep plays a role in determining the $\mathrm{L}-\mathrm{H}$ power threshold ${ }^{13}$.

Finally a power scan showed no clear effect on ELM size. The discharges became ELM-free as heating power approached the L-H transition power. In these cases, sawteeth were observed which dropped the stored energy by up to $20 \%$, but individual ELMs were not present.

\section{II.2. Lower single-nulls}

Single-null discharges exhibit the same secular density rise present in double-nulls, and as with the double-nulls, a minimum fueling rate is required to access H-mode. However routine Type I 
ELM activity is uncommon in NSTX single-null discharges. The results of a fueling rate scan on ELM behavior are shown in Fig. 4. At high fueling rates, rapid Type V ELMs were again observed. As the fueling rate was reduced to just above the minimum required for $\mathrm{H}$-mode access (panel 4b), giant ELMs (with $\square \mathrm{W} / \mathrm{W}_{0}$ up to $30 \%$ ) were observed. The physics of these large events is not well understood; stability calculations suggest the high-n ballooning mode as a candidate $^{5}$. For the Type V ELMs, an scan of the inner wall gap showed that the characteristics did not markedly change until the plasma was re-limited on the center stack, at which point an $\mathrm{H}$ L transition was observed.

\section{Type V ELM Characteristics}

The basic characteristics of the NSTX high performance, Type V ELM regime were shown in Fig. 1 of ref. 4 for a lower-single null diverted discharge with $\mathrm{I}_{\mathrm{p}}=0.8 \mathrm{MA}, \mathrm{B}_{\mathrm{t}}=0.5 \mathrm{~T}, \mathrm{P}_{\mathrm{NBI}}=4.1$ MW, elongation $\square \sim 1.9$, lower triangularity $\square_{\mathbb{L}} \sim 0.5$, and upper triangularity $\square_{U} \sim 0.3$. The line density continuously rose after the H-mode transition at $\mathrm{t}=230 \mathrm{~ms}$. Type V ELMs with a frequency $\sim 400 \mathrm{~Hz}$ were observed on the lower divertor $D_{\square}$ starting at $t \sim 340 \mathrm{~ms}$, although a few irregularly spaced Type V ELMs were observed near 290 and 320 ms. Gas puffing from the center stack continued throughout the discharge; the combination of this and the NBI fueling contributed to the observed density rise. The stored energy remained flat for about 370 ms or $7 * \square$, and the confinement enhancement over ITER-89P scaling ${ }^{14}$ was steady at $\sim 2.3$.

As mentioned previously, the Type V ELMs have no individual measurable impact on stored energy, but they are observed both in the radial profile of divertor $\mathrm{D}_{\square}$ emission, the ultra-soft $\mathrm{X}$ ray (USXR) diagnostic ${ }^{15}$, and a fast reciprocating probe introduced into the edge plasma. The 
ELMs increase the entire divertor $\mathrm{D}_{\square}$ profile by $~ 20-30 \%$, similar to the impact observed in the spatially integrated channels.

These ELMs are not the result of a persistent MHD mode. Low-n intermediate frequency (20-80 $\mathrm{kHz}$ ) coherent modes were observed in the core from 300-700 msec, but these modes started shortly after the Type V ELM activity. In other discharges it was observed that these coherent modes persisted even after the Type V ELMs stopped. Thus it is probable that these coherent modes are not the source of the ELMs, but rather a by-product of the conditions set-up in this high performance regime.

Nonetheless, the Type V ELMS have a distinct electromagnetic signature. An $n=1$ mode is observed in a toroidal Mirnov array below the outer midplane (Fig. 5) a few hundred microseconds before the USXR perturbation. The mode propagates in the counter plasma current direction and persists for $\sim 2$ toroidal transit times before dissipating (and re-appearing before the next USXR perturbation). The poloidal propagation of the mode is observed on a poloidal Mirnov array in the NSTX passive stabilizing plates and in the USXR system. The mode is first observed typically in the lower divertor and propagates to the outer midplane and then the top of the machine. The propagation down the inboard side of the machine is not observable due to diagnostic limitations, but the example in Fig. 5 re-appears in the lower divertor region and propagates upward again. The last temporal signature is the divertor $\mathrm{D}_{\square}$ rise, which occurs a few hundred microseconds afterwards. The characteristics of these ELMs have some similarities to observations of SOL current reported from conventional aspect ratio tokamaks ${ }^{16}$. 
One critical issue is the ability of these small ELMs to provide sufficient density and impurity control. Experimentally it is observed that the edge carbon density and radiation increases during these discharges, but the core radiated power and $\mathrm{Z}_{\text {eff }}$ remain low. Part of the density rise may occur due to continuous fueling from the center stack gas injection system, described earlier. The density rise from \#108729 was examined via a simple particle balance model $^{17}$;

the time dependence of the density was fit to obtain $\square_{p}^{*} \sim 0.5 \mathrm{~s}$, yielding a $\square_{p}^{*} / \square_{E} \sim 9$. We note that this value is approximately the same value obtained in DIII-D long pulse H-modes before active divertor pumping was enabled; the DIII-D ratio was reduced $\sim 50 \%$ (by reducing $\square_{p}^{*}$ ) with efficient in-vessel cryo-pumping (i.e. line average density was reduced by at least a factor of 2 ) $^{18}$. We also note that the density rises even more quickly in NSTX ELM-free discharges than in discharges with Type V ELMs. Thus it is probable that NSTX could achieve a similar reduction in $\square_{p}^{*} / \square_{E}$ with an active pumping scheme of similar efficiency, implying that the particle confinement reduction provided by these ELMs will allow for sufficient density control.

\section{Summary, Discussion and Conclusions}

Many different ELM types have been observed in NSTX, including conventional Type I, Type III, a new small ELM (which we designate as Type V), and 'giant' ELMs. The Type I ELMs reduce the plasma stored energy by up to $10 \%$, and the pedestal energy by up to $30 \%$. The size of these Type I ELMs decreases with density and as the magnetic configuration is shifted from a double-null to a lower-single null. Giant ELMs are observed under low recycling conditions in lower-single null configuration and can decrease the plasma stored energy by up to $30 \%$. The Type V ELM regime is observed in high density, high performance discharges. The Type V 
ELMs have an electromagnetic signature of a short-lived $n=1$ mode rotating counter to the plasma current, and are observed in a poloidal Mirnov array and the USXR, as well as the divertor $\mathrm{D}_{\square}$ profile. Particle confinement estimates indicate that efficient in-vessel pumping would probably allow sufficient density control for current drive scenarios.

The NSTX Type V regime appears to be distinct from the DIII-D quiescent H-mode, which requires an edge harmonic oscillation for particle control. The NSTX Type V regime is also distinct from the PDX forced density rise scenario and the Alcator C-MOD Enhanced $\mathrm{D}_{\square} \mathrm{H}$ mode, which require an edge quasi-coherent mode for particle control. Experiments to determine the underlying physical mechanisms for Type V ELMs, as well as to test the extrapolability to low collisonality conventional aspect ratio tokamaks are being considered.

\section{Acknowledgements}

This research was supported by the U. S. Dept. of Energy under contracts DE-AC0500OR22725, DE-AC02-76CH03073, and W-7405-ENG-36, and grants DE-FG03-95ER54294, DE-FG02-99ER54519, DE-FG02-99ER54523, and DE-FG02-99ER54524, We gratefully acknowledge the contributions of the NSTX operations staff.

This work was performed under the auspices of the U.S. Department of Energy by the University of California, Lawrence Livermore National Laboratory under Contract No. W-7405-Eng-48. 


\section{Figure Captions}

1. Different ELM types observed in NSTX: (a) Type I, (b) Type III, (c) new, Type V, and (d) mixed Type V with a 'giant' ELM.

2. Flow rate scan in double-null configuration: (a) 39 Torr-1/s average flow, (b) 14 Torr-1/s, (c) 9 Torr-1/s, and (d) 3 Torr-1/s.

3. Type I ELM size scaling: (a) ELM loss size vs. $\overline{n_{e}}$, (b) ELM energy loss fraction vs. $\overline{n_{e}}$, (c) ELM pedestal energy loss fraction vs. $\overline{n_{e}}$, (d) ELM frequency vs. $\overline{n_{e}}$. (e) ELM loss size vs. frequency, and (f) ELM loss size vs. $\operatorname{drsep}$ (see text).

4. Fueling scan in LSN (a) 39 Torr-1/s average flow (b) 14 Torr-1/s, and (c) 11 Torr-1/s.

5. Magnetic signature of Type V ELMs: (a) divertor $D_{\square}$, (b) magnetic perturbation size at wall at $\square=30^{\circ}$, (c) toroidal Mirnov array with dark blue bands showing mode toroidal propagation counter to $I_{p}$, and (d) passive plate Mirnov array with dark blue bands showing mode poloidal propagation upward. The approximate mode birth is highlighted by circles in (c) and (d). 
Figure 1

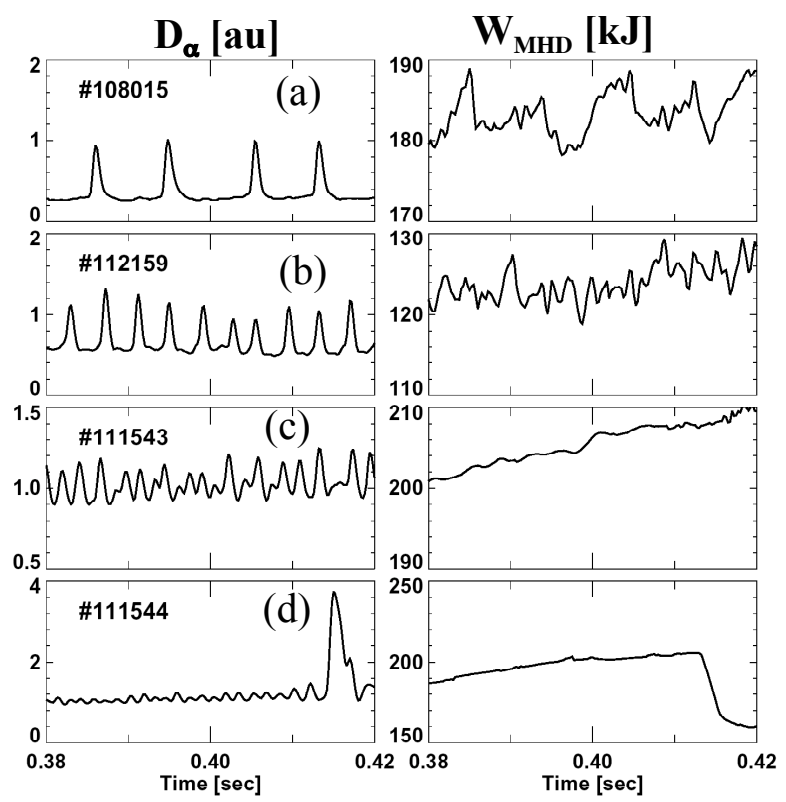


Figure 2

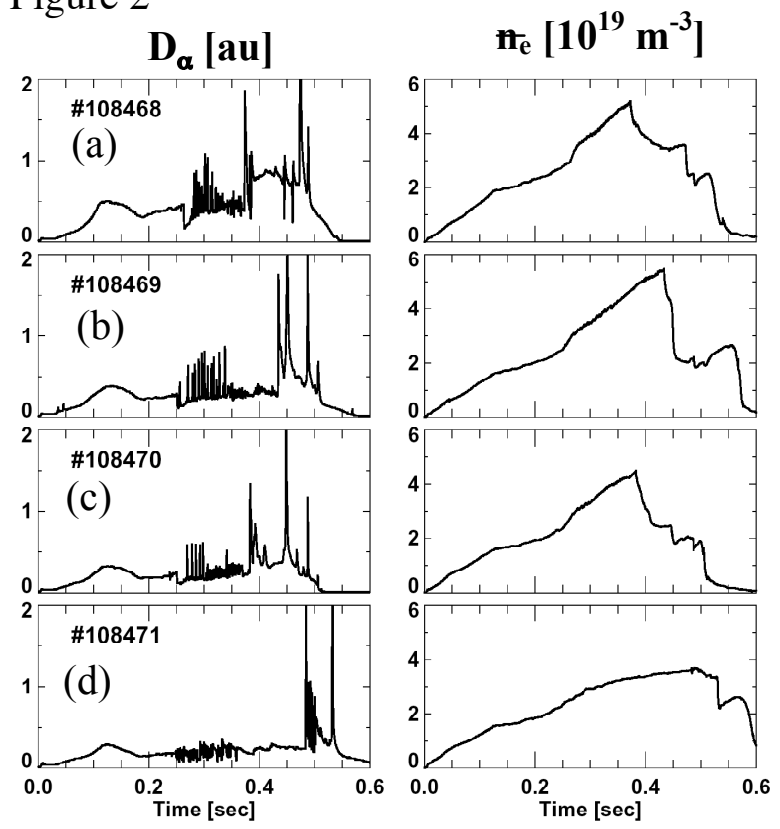


Figure 3
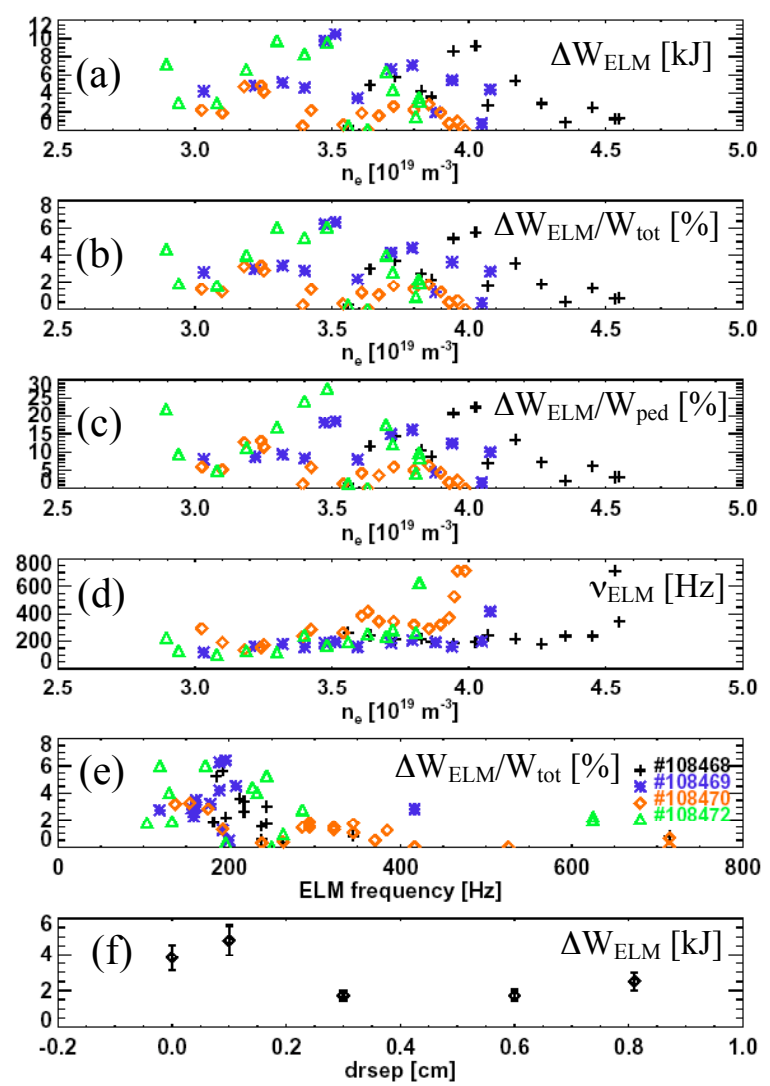
Figure 4

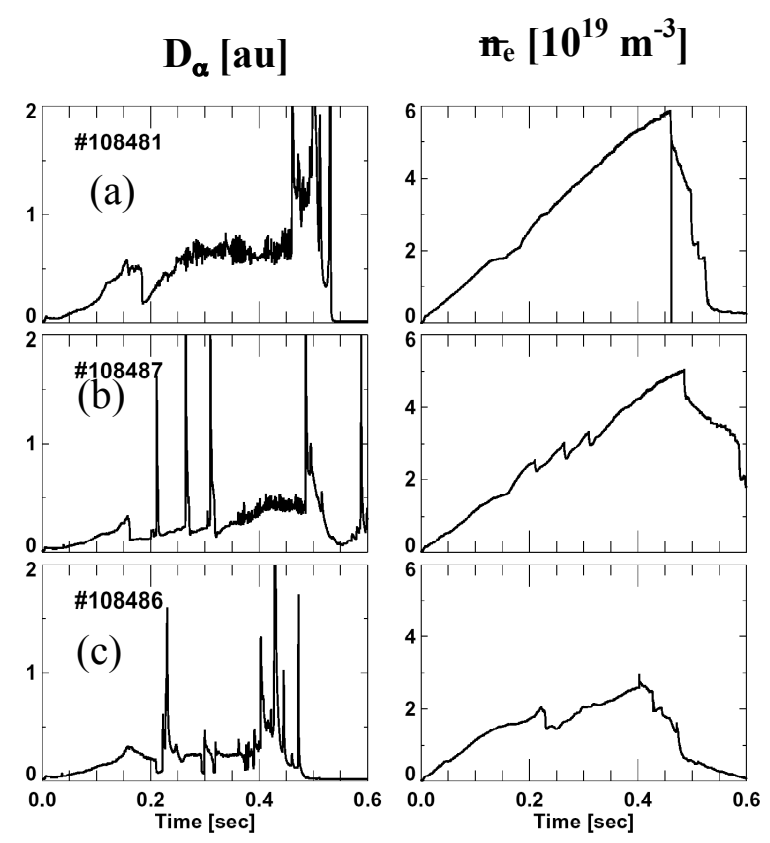


Figure 5
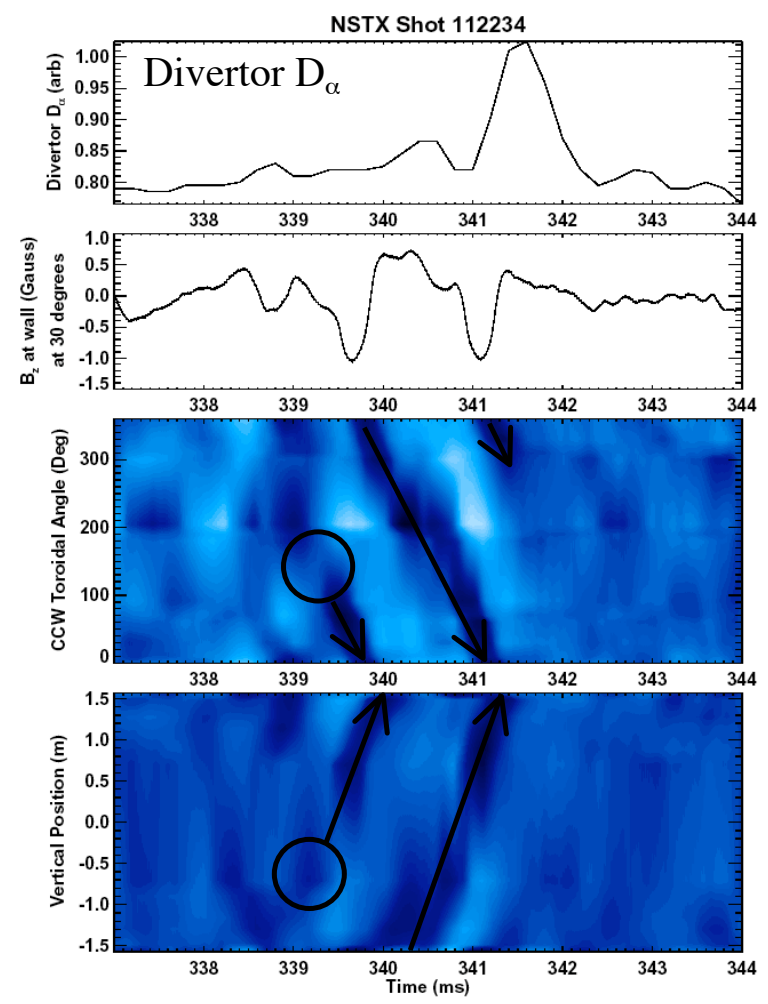


\section{References}

$1 \quad$ I. P. B. Authors, et. al., Nuclear Fusion 39, (1999)2137.

A. Loarte, et. al., J. Nucl. Materials 313-316, (2003)962.

M. Ono, et. al., Nuclear Fusion 40, (2000)557.

R. Maingi, et. al., Physical Review Letters submitted, (2004).

C. E. Bush, et. al., Physics of Plasmas 10, (2003)1755.

L. L. Lao, et. al., Nuclear Fusion 25, (1985)1611.

S. A. Sabbagh, et. al., Nuclear Fusion 41, (2001)1601.

J. G. Cordey, et. al., Nuclear Fusion 43, (2003)670.

R. Maingi, et. al., Nuclear Fusion 43, (2003)969.

R. Maingi, et. al., Plasma Physics Controlled Fusion 46, (2004)A305.

A. W. Leonard, et. al., Journal of Nuclear Materials 290-293, (2001)1097.

O. Gruber, et. al., Proc. of the 19th International Conference on Plasma Physics and

Controlled Fusion, Lyon, 2002 (IAEA, Vienna), (2002)paper EX/C2.

13 H. Meyer, et. al., Plasma Physics Controlled Fusion 46, (2004)A291.

$14 \quad$ P. N. Yushmanov, et. al., Nuclear Fusion 30, (1990)1999.

15 D. Stutman, et. al., Review of Scientific Instruments 74, (2003)1982.

16 H. Takahashi, et. al., Nuclear Fusion at press, (2004).

17 G. L. Jackson, et. al., J. Nucl. Materials 196-198, (1992) 236.

18 M. A. Mahdavi, et. al., J. Nucl. Materials 220-222, (1995)13. 
Univverilty of California

Lawrence Livermere National Laboratery

Technical Information Department

Livermore, CA 94551

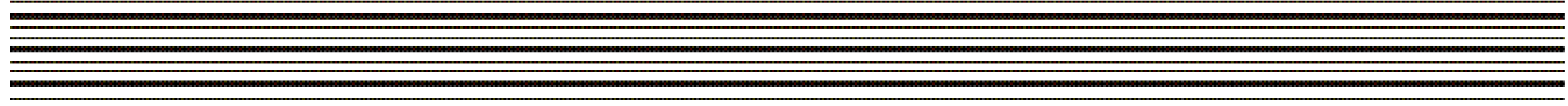

